\title{
Influence of Domain Structure on The Mechanical Properties of Thermoplastic Polyurethane Materials
}

\author{
Tomoe Sunada, Mizue Kuriyagawa, \\ Takanobu KaWAMURA, and Koh-hei NitTA* \\ Division of Material Engineering, Graduate School of Natural Science and Technology, Kanazawa University, \\ Kakuma, Kanazawa, Ishikawa 920-1192 Japan \\ *Corresponding author: nitta@t.kanazawa-u.ac.jp \\ Received August 30, 2011; Accepted October 18, 2011 \\ (c) 2011 The Society of Rubber Industry, Japan
}

\begin{abstract}
The tensile deformation of thermoplastic polyurethanes (TPUs) with varying sizes of hard domain was investigated to produce a new form of constitutive equation. The equation is expressed using the plastic component of the deformation of the hard domains and a network component based on van der Waals' equation. A TPU formulation with a large hard domain shows a higher stress level and higher stress-strain gradient. It was found that the stress level is dominated by the plastic component, resulting from the stiffness of the hard domains and the stress gradient is determined by the network component, resulting from the extension of the soft segment chains between the hard domains.
\end{abstract}

Keywords Polyurethane, Tensile properties, Constitutive equation, Morphology.

\section{Introduction}

Elastomeric products such as tires, rubber tape, packing, and anti-vibration mountings play an essential role in daily life and require high performance materials. In addition, the demand for synthetic elastomers is expected to increase with the growing demand for automobile tires. In general, recycling vulcanized rubbers is difficult compared to thermoplastic materials because chemical cross-linking does not allow the material to be simply melted and reformed. The multiple stage vulcanizing process and the addition of strengthening fillers required to transform raw rubber into useful materials are effectively irreversible processes. Thermoplastic elastomers such as thermoplastic polyurethanes (TPU) do have a considerable advantage in that they are capable of being recycled by simply being melted and reformed directly into new articles.

TPU based materials are an important elastic materials with useful mechanical characteristics. ${ }^{1-6)}$ The modulus and hardness of TPUs lie between vulcanized rubber and conventional thermoplastic polymers. TPU is composed of a soft component (soft domains) and molecular confinement component (hard domains) which act in the same fashion as chemical cross-links giving rubber-like (eg large reversible strain) bulk properties. ${ }^{7-9}$ Since there is no need chemically cross-link (vulcanize) to produce these properties, TPUs can be used in conventional plasticprocessing methods such as injection molding, extrusion, and blow molding. The significant difference between a vulcanized rubber and a TPU is in the structure of the cross-linking arrangement: vulcanized rubbers are crosslinked by chemical bonds whereas the cross linking structure of TPU is by physical interaction between crystallites and glassy aggregates. In terms of mechanical properties this means that when compared to vulcanized rubbers, TPU materials show plastic deformation at high strains and highly elastic properties at smaller strains. In the future, the market for TPU products is expected to grow because of their perceived advantages in both processability and recyclability.

TPUs containing urethane bonds are considered to be multi-block copolymers, and to be composed of flexible chain soft segments and hard segments characterized by lower molecular mobility. ${ }^{7,8)}$ The hard segments form domains by hydrogen bonding between the urethane bonds within the hard segments, and the hard domains act as pseudo-cross-links. The shape and size of the hard domains both play a central role in the bulk mechanical performance of the material, and can be controlled by the molecular weight and molecular composition of the raw materials from which the polymers are made. The paper describes an investigation into the influence of these cross- 


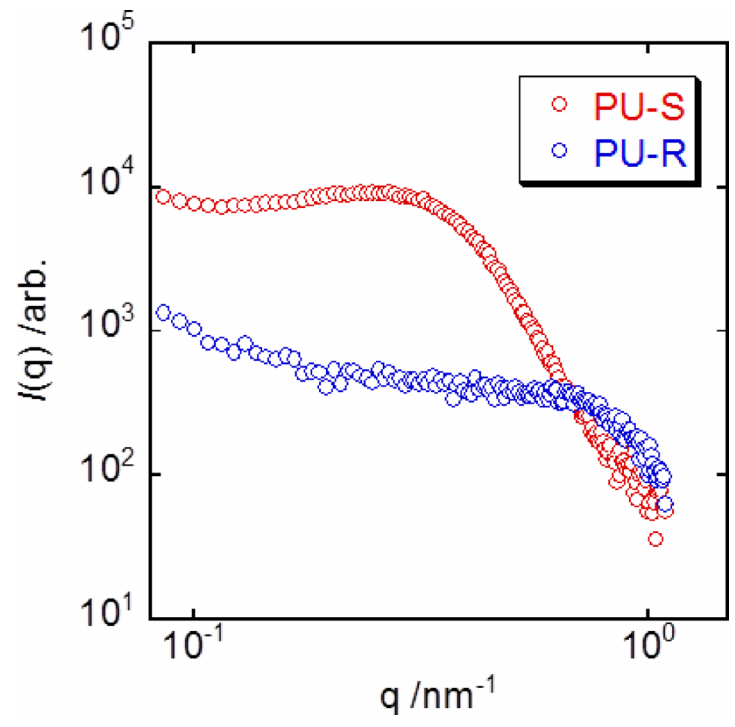

Figure 1. Scattering profiles of SAXS.

link structures on the tensile properties of several TPU materials with different domain structures. This provides an insight into the influence of polymer microstructure on the bulk stress-strain behavior of TPUs.

\section{Experimental}

\section{Materials}

We used two commercial flexible thermoplastic polyurethane foam formulations known to have different domain structures: one has sphere domains (PU-S) and the other has rod-like domains (PU-R) Each polyurethane foam formulation was prepared using toluene diisocyanate (TDI) for hard segment formation, multifunctional glycol as a chain extender, and polyether for soft segment formation.

The two foam samples were then completely melted at $210^{\circ} \mathrm{C}$ (PU-S) and $195^{\circ} \mathrm{C}$ (PU-R), by being placed between two aluminum plates in a laboratory hot press for $10 \mathrm{~min}$ and pressed at $200 \mathrm{MPa}$ for $5 \mathrm{~min}$. On removal from the press, the samples were slowly cooled to room temperature. The resulting compression molded films were about $300-350 \mu \mathrm{m}$ thick.

\section{Characterization}

Small angle X-ray scattering: The SAXS measurements were performed on beam line 10C at the Photon Factory, Japan. The samples were irradiated with monochromatized (wavelength, $\lambda=0.1488 \mathrm{~nm}$ ) X-rays radiated from the synchrotron. The scattered X-rays were detected by a onedimensional position sensitive proportional counter (PSPC). The SAXS profiles for two samples are shown in

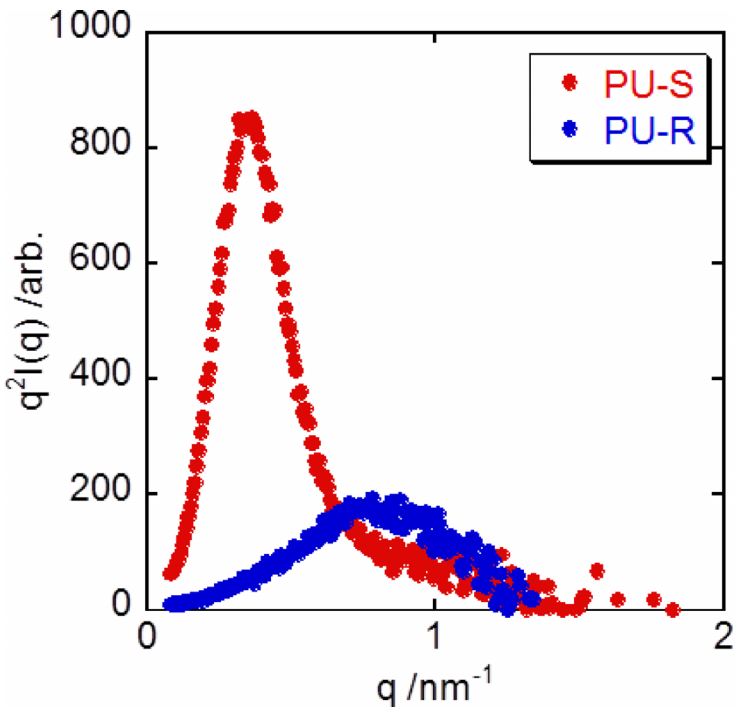

Figure 2. Kratky plots of SAXS.

Figure 1. The Kratky plots shown in Figure 2, indicate by the distinct bilaterally symmetric peak at $\mathrm{q}=0.33 \mathrm{~nm}^{-1}$, that there are spherical formations in PU-S. The broader peak at $\mathrm{q}=0.80 \mathrm{~nm}^{-1}$, indicates that cylindrical formations are present within the PU-R sample and the cylinder size is estimated to be about $4.8 \mathrm{~nm}$. The radius of the PU-S spheroids was estimated from the peak position in Kratky plots and Bragg's equation, to be about $19 \mathrm{~nm}$.

Dynamic mechanical analysis: The dependence on temperature of viscoelasticity was measured over a temperature range of -100 to $150^{\circ} \mathrm{C}$ using a DVE-V4 (Rheology Co.) instrument. Rectangular specimens $(20 \times$ $5 \mathrm{~mm}$ and $0.3 \mathrm{~mm}$ thick) were used, and the measurements were carried out at constant frequency of $10 \mathrm{~Hz}$ with a fixed amplitude of $2 \mathrm{~mm}$. The tensile storage modulus $E^{\prime}$, loss modulus $E^{\prime \prime}$, and loss tangent $\tan \delta$ were recorded at a heating rate $2^{\circ} \mathrm{Cmin}^{-1}$ under a nitrogen atmosphere. The temperature dependence of $E^{\prime}$ and $E^{\prime \prime}$ is shown in Figure 3. The molecular weight between cross-links estimated from the value of $E^{\prime}$ in the rubbery plateau region and the glass transition temperature was estimated from the peak temperature in $E^{\prime \prime}$. These results are summarized in Table 1.

Tensile tests: to investigate the dependence of mechanical properties on the sample structure, tensile tests were performed using a compact tensile tester with a specially designed and constructed heated specimen chamber. Dumb-bell shaped tensile test specimens with width of $4 \mathrm{~mm}$ and a gauge length of $10 \mathrm{~mm}$ were used. The tensile tests were performed at a fixed crosshead speed of $10 \mathrm{~mm} / \mathrm{min}$ at room temperature $\left(25^{\circ} \mathrm{C}\right)$ and then from $T_{\mathrm{g}}$ to $T_{\mathrm{g}}+40^{\circ} \mathrm{C}$. The details of the test conditions are 


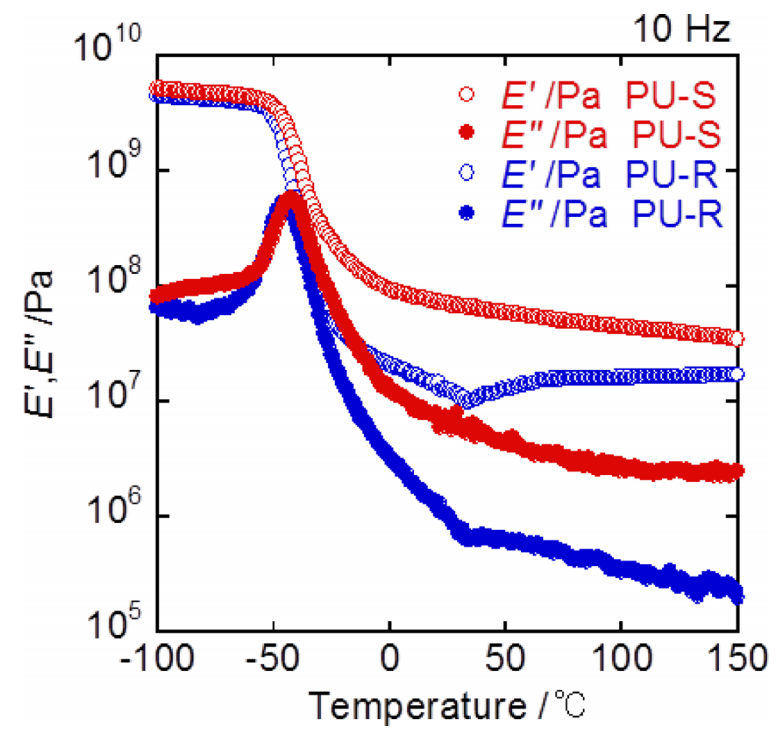

Figure 3. Temperature dependences of storage elastic modulus $E^{\prime}$ and loss elastic modulus $E^{\prime \prime}$.

Table 1. Glass transition temperature $T_{\mathrm{g}}$ and molecular weight between cross-links $M_{\mathrm{e}}$

\begin{tabular}{ccc}
\hline & $T \mathrm{~g} /{ }^{\circ} \mathrm{C}$ & $M_{e}$ \\
\hline PU-S & -42 & 200 \\
PU-R & -45 & 620 \\
\hline
\end{tabular}

Table 2. Uniaxial tensile tests at varying temperatures.

\begin{tabular}{ccc}
\hline & PU-S $\left(T_{\mathrm{g}}-42^{\circ} \mathrm{C}\right)$ & PU-R $\left(T_{\mathrm{g}}-45^{\circ} \mathrm{C}\right)$ \\
\hline$T_{\mathrm{g}}$ & -42 & -45 \\
$T_{\mathrm{g}}+10^{\circ} \mathrm{C}$ & -32 & -35 \\
$T_{\mathrm{g}}+20^{\circ} \mathrm{C}$ & -22 & -25 \\
$T_{\mathrm{g}}+30^{\circ} \mathrm{C}$ & -12 & -15 \\
$T_{\mathrm{g}}+40^{\circ} \mathrm{C}$ & -2 & -5 \\
\hline
\end{tabular}

summarized in Table 2.

\section{Theoretical considerations}

From the shape and size of the hard domains obtained from the SAXS profiles and the molecular weight $M_{\mathrm{e}}$ (estimated from viscoelastic data) between adjacent junctions of the samples, we can devise a molecular structure model for PU-S and PU-R-see Figure 4. We then present a constitutive relation validated against real elastomeric materials based on the fact that the cross-links or junctions in elastic network have a finite volume and that mutual molecular interactions exist. The constitutive relation of an ideal elastomer having no inter-chain interactions corresponds to the state equation of an ideal gas. Whereas TPU has significant mutual interactions
PU-S

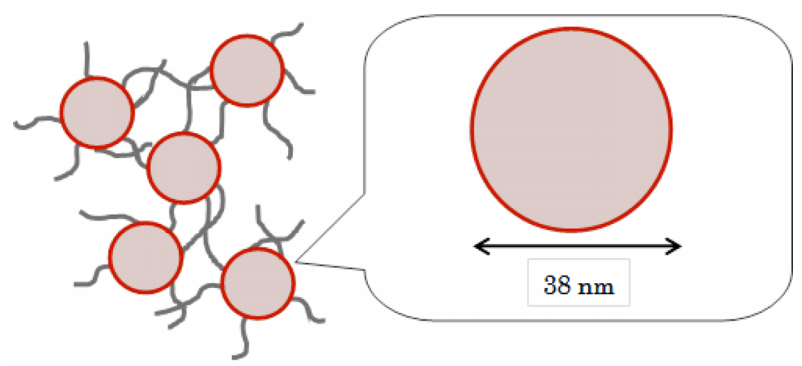

PU-R

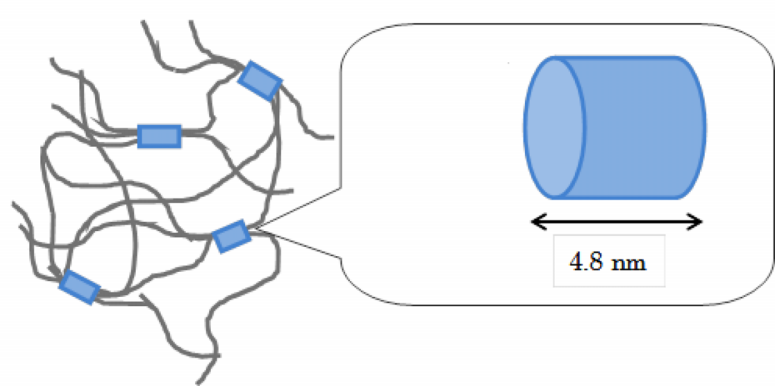

Figure 4. Schematic illustrations of molecular structure for PU-S and PU-R.

between the chains and/or junctions that are described by a van der Waals' equation to include attractive and repulsive interactions.

It is known that the stress-strain behavior of TPU elastomers exhibits both elastic and plastic responses. ${ }^{1}$ Theoretical considerations of an ideal elastomer, indicate that the origin of elastic behavior is through the recovery of conformational entropy, and it has been assumed that there are no mutual interactions between the molecular network chains. However, in the case of the actual PU samples, a finite volume of hard domains act as junctions and mutual interaction between the chains is present.

Kilian ${ }^{10,11)}$ proposed a real network model where cohesive interaction and excluded volume effects on the free energy of a Gaussian network are taken into account. Statistical thermodynamic calculations indicate that the relationship between load $f$ and extension ratio $\lambda$ under uniaxial elongation results in

$$
f=\frac{N k T / L_{0}}{\left(\frac{1}{\Psi^{\lambda}}-b\right)}-a_{0}\left(\Psi^{\lambda}\right)^{2}
$$

where $L_{0}$ is the initial length of the specimen, $k$ is the Boltzmann constant, $N$ is the number of network chains, $b$ is the repulsive force between crosslinks, $a_{0}$ is the attraction force. The deformation function $\psi$ is given by $\left(I_{1}-3\right) / 2$ using the first strain invariant $I_{1}$ and the $\psi^{\lambda}$ is the derivative of $\psi$ with respect to $\lambda$, resulting in $\lambda-\lambda^{-2}$ for 


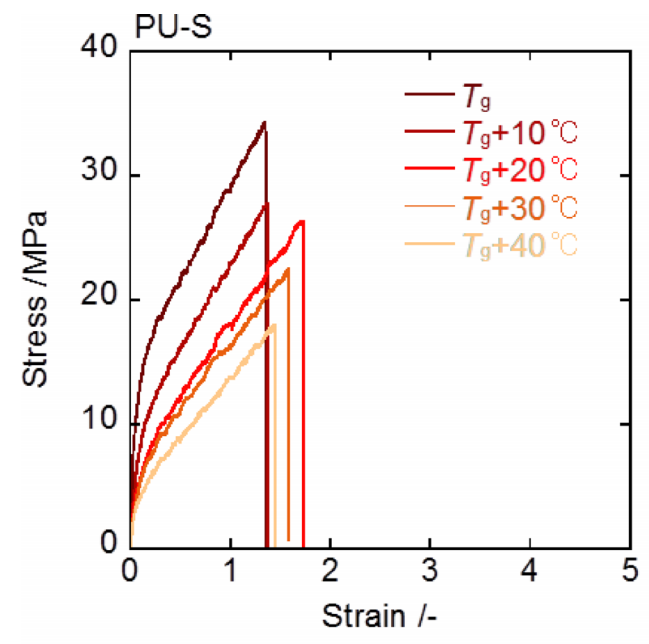

(a)

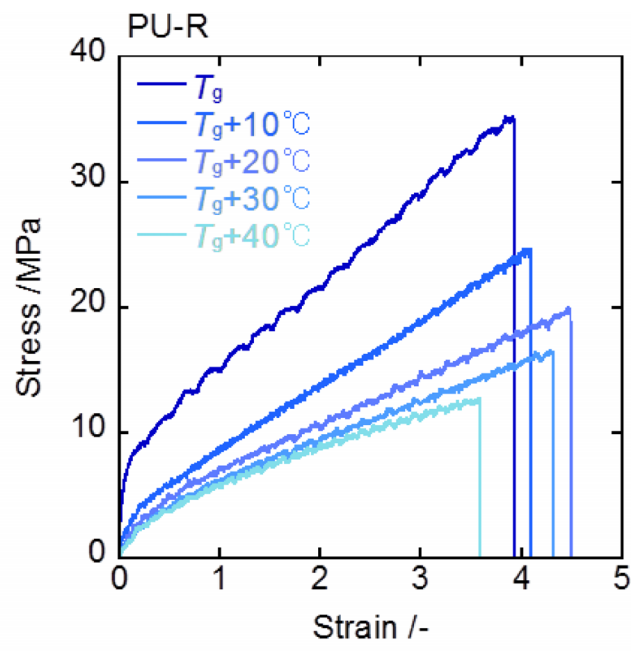

(b)

Figure 5. Stress-strain curves for PU-R and PU-S with different temperatures.

uniaxial extension ${ }^{11)}$. Using the parameters $3 G_{e}=$ $(d f / d \lambda)_{\lambda \rightarrow 1} / A_{0}=k N T / V_{0}$, where $A_{0}$ and $V_{0}$ are the initial area and volume of the specimen, and $a=a_{0} L_{0} /(N k T)$, the stresselongation curve for the network component has the form:

$$
\sigma=G_{e}\left\{\frac{\Psi^{\lambda}}{1-\Psi^{\lambda} b}-a\left(\Psi^{\lambda}\right)^{2}\right\}
$$

where $\sigma$ is the nominal stress. According to Eq.(2), the force becomes infinite if the elongation $\Psi^{\lambda}$ approaches $b$, indicating that the inverse of the parameter $b$ is related to the ultimate elongation $\lambda_{\max }$ between adjacent junction points.

Due to hydrogen bonding, hard segment chains of PU lie parallel to one another in a fringed micelle structure. Therefore, plastic flow around the hard segments can be considered to occur during a tensile deformation, even below the melting or glass transition temperatures. The strain energy function of the hard segment is modeled here through finite deformation of hard polycrystalline materials using Leonov's approach. ${ }^{12}$ ) This introduces the strain energy function of polycrystalline hard materials for semicrystalline polymer solids to describe plastic flow behavior where the stacking crystalline phase acts as a cross-link. Macroscopic deformation behavior of our TPU samples is assumed to be isotropic because of the random distribution of the hard domains. Then the strain energy $y_{H}$ can be expressed as a function of the second invariant $I_{2}$, giving $\Psi_{\mathrm{H}}=G_{p}\left(I_{2}-3\right) / 2$ where $G_{p}$ becomes the plastic flow stress for the component. The stress given by $\mathrm{d}_{\Psi_{H}} / \mathrm{d} \lambda$ rapidly reaches a constant value $G_{p}$, we call this the "plastic component". Previously, G'Sell et al. ${ }^{13)}$ found that the constitutive equations for various semicrystalline polymers can be described by the sum of the ideal elastic component and the plastic deformation component. Here we propose a constitutive relation for TPU materials by the extension of their constitutive models using Kilian's real network model in the form of the addition of both equations:

$$
\sigma=\frac{E_{p}}{3}\left(1-\frac{1}{\lambda^{3}}\right)+\frac{E_{e}}{3}\left(\frac{\Psi^{\lambda}}{1-\Psi^{\lambda} b}-a\left(\Psi^{\lambda}\right)^{2}\right)
$$

where $E_{\mathrm{e}}=G_{\mathrm{e}} / 3$ is the mechanical parameter for contribution of the network component, and $E_{\mathrm{p}}=G_{\mathrm{p}} / 3$ is that of the plastic component. We assume that the PU materials are incompressible, i.e. $I_{3}=0$. The second term contributes to the slope of stress-strain curve and the first term determines the overall stress level.

\section{Results and Discussion}

The stress-strain curves measured at temperatures ranging from $T_{\mathrm{g}}$ to $T_{\mathrm{g}}+40^{\circ} \mathrm{C}$ and at room temperature $\left(25^{\circ} \mathrm{C}\right)$ for PU-S and PU-R are summarized in Figure 5. It was found that the shape of stress-strain curves above $T_{\mathrm{g}}$ for both samples is almost the same and that the breaking strains are independent of temperature. The stress level of both PU-S and PU-R decreases with increase in temperature. The breaking point and the slope of the stress-strain line at higher strains are much reduced for PU-S compared to those of PU-R. Figure 6 summarizes the Young's modulus and the toughness estimated from the stress-strain curves, as a function of temperature. The Young's modulus exponentially decreased to a fixed equilibrium value whilst the toughness linearly decreased 


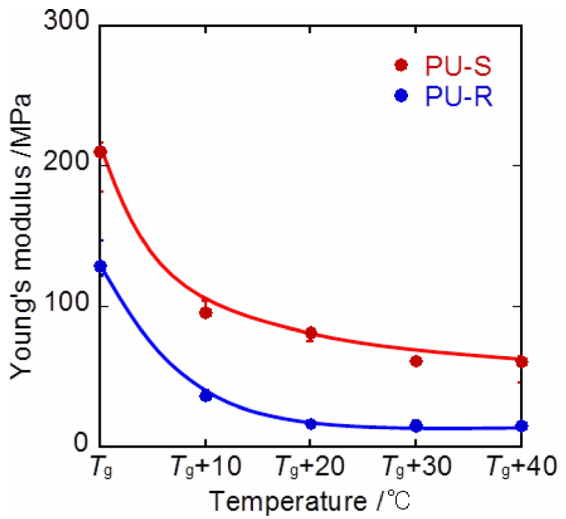

(a)

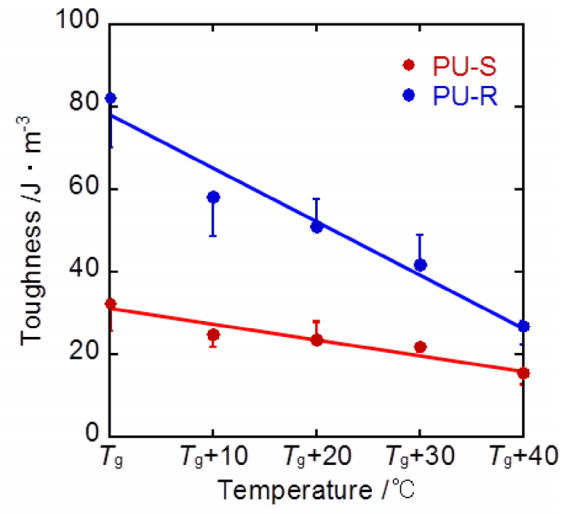

(b)

Figure 6. Young's modulus and toughness vs. temperature.

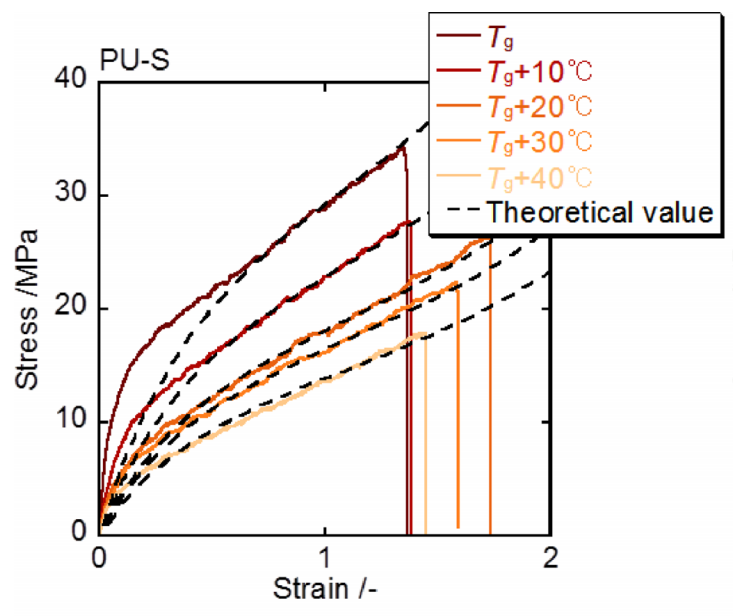

(a)

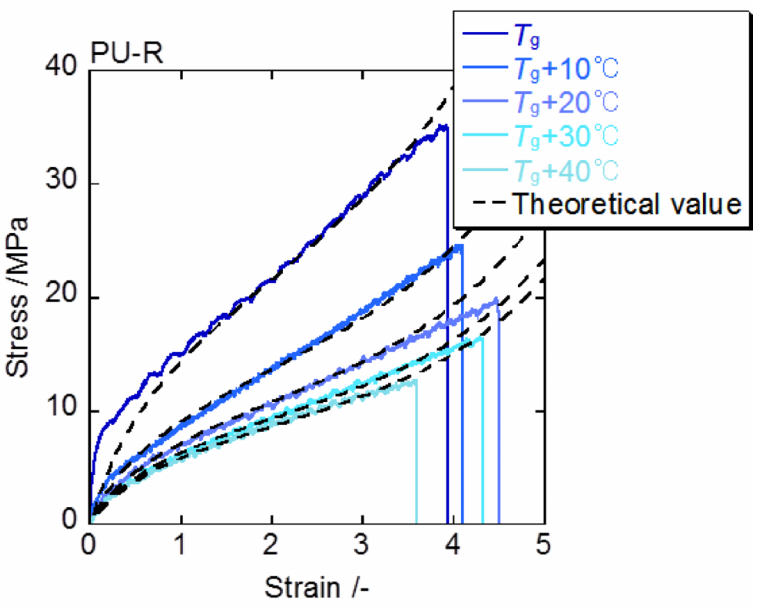

(b)

Figure 7. Comparison of theoretical predictions of stress-strain curves with experimental values.

as the temperature increased.

The theoretical constitutive equation Eq.(3) is fitted to the experimental stress-strain curves to obtain certain mechanical parameters such as the attractive interaction parameter $a$, the repulsive interaction parameter $b$, the mechanical parameters of the elastic component $E_{e}$, and the mechanical parameters of the plastic component $E_{p}$. Since parameter $b$ is a structural parameter directly related to the finite length of the molecular chains between adjacent hard domains, the optimization process was performed with the assumption that $b$ is independent of temperature. A relatively good fit of the theoretical equation to the experimental data was obtained except for material PU-S in the initial strain regions, and in particular when approaching $T_{g}$ - see Figure 7 .

Figure 8 shows the temperature of the interaction parameter $a$ for both samples, suggesting that the parameter $a$ is an intrinsic parameter based on the molecular structure and structural morphology of the samples. The parameter $a$ for PU-S is larger than that of PU-R, indicating a stronger attractive inter-domain interaction than is present in PU-S. This is because PU-S has larger hard domains and shorter distances between the hard domains than PU-R.

As previously described, theory indicates that the inverse of parameter $b$ is given by, which is approximately equal to $\lambda_{\max }$, because $\lambda_{\max }$ is large. The maximum extension for the Gaussian network is approximately proportional to the square root of the molecular weight $M_{e}$ between adjacent junction points. Consequently, the present theory predicts the following relation:

$$
\frac{b^{P U-S}}{b^{P U-R}}=\sqrt{\frac{M_{c}^{P U-R}}{M_{c}^{P U-S}}}
$$




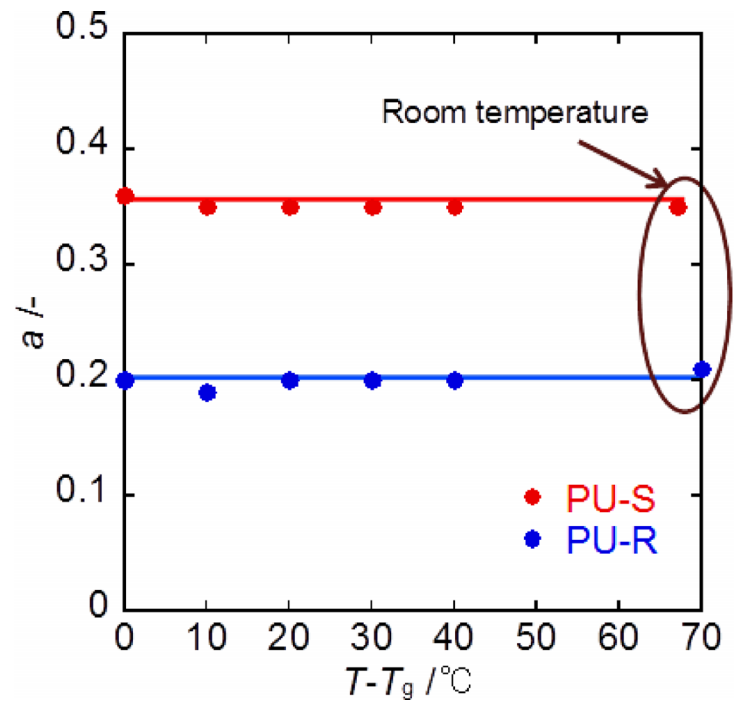

Figure 8. Interaction parameter $a v s$. temperature.

Table 3. $M_{c}, \lambda_{\max }$ and parameter $b$.

\begin{tabular}{cccl}
\hline & $M_{c} /-$ & $\lambda_{\max } /-$ & $b /-$ \\
\hline PU-S & 200 & 6.5 & 0.15 \\
PU-R & 620 & 11 & 0.091 \\
\hline
\end{tabular}

where the superscript denotes the sample code. Table 3 shows parameter $b$, the molecular weight $M_{e}$ between cross-links, and the maximum extensional ratio. The values of $M_{e}$ were estimated from the rubber plateau at the maximum point on the dynamic mechanical spectra and the maximum extension ratios were estimated from the fitting parameter $b$. There is close agreement with the experimental data shown in Table 3. The maximum extension ratio $\lambda_{\max }$ is larger than the actual break extension. The strong upward curvature at high extensions in the theoretical curves is a consequence of limited chain extensibility ${ }^{10)}$. The actual break takes place before this increase in gradient of the curve and this may be caused by the strong repulsive effects between the bulky hard domains when these domains move closer during extension. However, it is interesting to note that the ratio of actual $\lambda_{\text {break }}$ between PU-S and PU-R is almost the same with the ratios of $\lambda_{\max }$.

In addition, the $E_{\mathrm{p}}$ of the plastic component values also deceases with increasing the temperature. PU-S having larger hard domains, shows a higher $E_{\mathrm{p}}$ but both $E_{\mathrm{p}}$ values ultimately reduced to the same equilibrium value (see Figure 9) as temperature increases. This suggests that the dominant factor for $E_{\mathrm{p}}$ is the stiffness of the hard domains as they act as network junctions and that a rise in temperature softens the hard domains, resulting in the

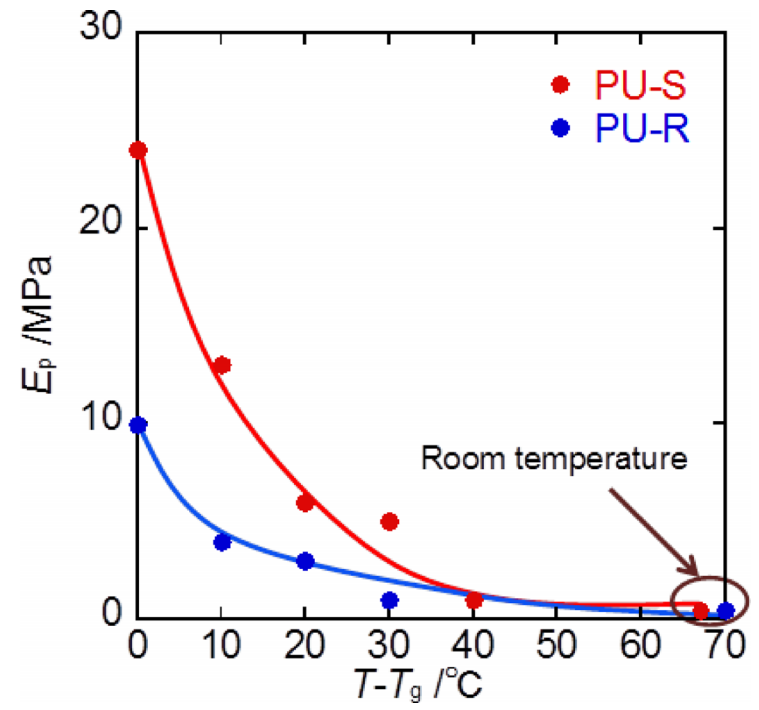

Figure 9. Young's modulus $E_{\mathrm{p}} v s$. temperature.

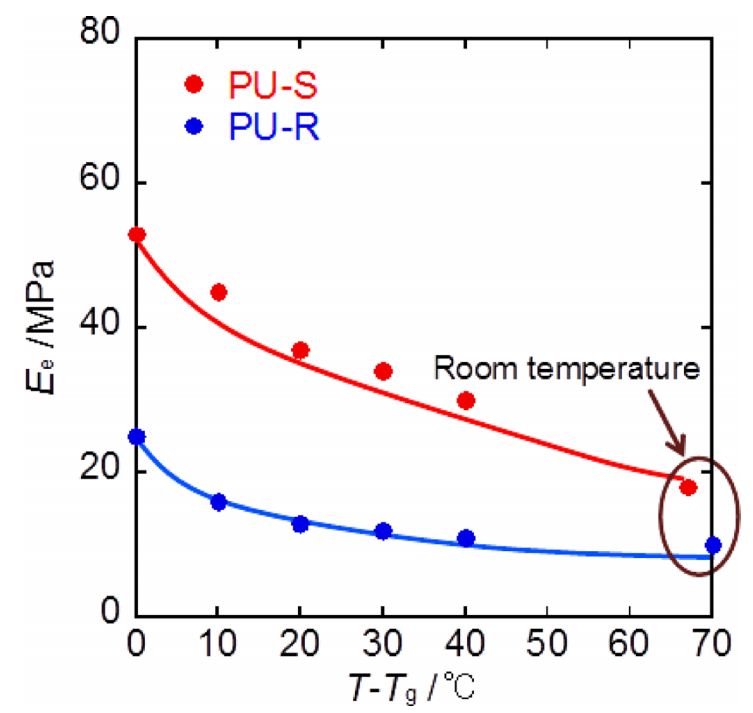

Figure 10. Young's modulus $E_{\mathrm{e}} v s$.temperature.

observed decrease in the plastic component to a constant value.

The network modulus $E_{\mathrm{e}}$ of PU-S and PU-R are plotted against temperature in Figure 10. It was found that $E_{\mathrm{e}}$ decreases with increase in temperature for both materials. The entropic modulus shows a positive dependence on temperature whereas the network modulus monotonically decreases as temperature increases.

This suggests that the value of $E_{\mathrm{e}}$ is associated with the molecular mobility of the soft-segments around the hard domains. A rise in temperature causes the release of the soft segments formerly restricted by the hard domains; in other words, the segment number between the hard domains is apparently decreased as the temperature 
approaches $T_{g}$.

It is interesting to note that there is no great difference between the ratios of $E_{e}$ to $E_{e}+E_{p}$ between PU-S and PU$\mathrm{R}$ although the absolute values of these moduli are both larger for PU-S due to the presence of large hard domains. This indicates that the fraction of the contribution from the network to overall tensile behavior is the same with both TPU samples.

\section{Conclusions}

We have investigated how the domain structures of thermoplastic polyurethane materials affect the mechanical properties above the glass transition temperature. Two thermoplastic polyurethanes (PU-R and PU-S) known to have different domain structures were investigated: PU-R has cylindrical hard domains about 3-4 nm long, and PU-S has spheroidal domains of about $40 \mathrm{~nm}$ diameter. The stress-strain curves of these polyurethane materials are composed of two components: the plastic component expressed by the second invariant which determines the overall stress level, and the network component which dominates the stress gradient at higher strains. The plastic component is controlled by the stiffness of the hard domains and the network component is controlled by the deformation of the soft segments between the hard domains. The stress levels of the stress-stain curves for both materials decreased with increasing temperature. This is because of the reduction in the contribution from the plastic component caused by the softening of the hard domains. The increasing stress gradient at higher strains is determined by the elastic deformation of the network component. The finite length of the soft segments between adjacent hard domains (junction points) predominantly affects the mechanical response of the network component.

It is well known that thermoplastic polyurethane materials exhibit both plastic and elastic deformation. In this paper, we present an analytical method for determining the mechanical response of materials having such characteristics. The method has wide applicability for understanding the tensile behavior of thermoplastic materials, as well as for strain-induced crystallization and phase separation.

\section{References}

1) Petrovic Z., Ferguson J.: Prog. Polym. Sci., 16, 695 (1991).

2) Cooper S.C., Tobolsky A.V.: J. Appl. Polym. Sci., 10, 1837 (1966).

3) Seymour R. W., Este C. M., Cooper S. C.: Macomolecules, 3, 579 (1970).

4) Furukawa F., Mitusi Y., Fukumaru T., Kojio K.: Polymer, 46, 10817 (2005).

5) Kojio K., Furukawa M., Motokucho S., Shimada M., Sakai M., Macromolecules, 43, 8322 (2009).

6) Ng H. N., Allegrezza, A. E., Seymour R. W., Cooper S. L.: Polymer, 14, 255 (1973).

7) Bonart R.: J. Macromol. Sci. Phys. B2, 115 (1968)

8) Bonart R.: J. Macromol. Sci. Phys. B3, 337 (1969)

9) Kimura I., Kshikawa H., Ono H., Yoshihara N., Nomura S., Kawai H., Macromolecules, 7, 355 (1974).

10) Kilian H.-G.: Polymer, 22, 209 (1981)

11) H.-G. Kilian, Colloid Polym. Sci. 263, 30 (1985).

12) A. I. Leonov, Int. J. Solids Structure, 39, 5913 (2002).

13) C. G'Sell, J. J. Jonas, J. Mater. Sci., 14, 583 (1979). 